\title{
A Construction Robot for Autonomous Plastering of Walls and Ceilings
}

\author{
Johan Forsberg Roger Aarenstrup Åke Wernersson \\ Robotics and Automation
Luleå University of Technology, S-971 87 Luleå, Sweden \\ Email: robotics@sm.luth.se
}

\begin{abstract}
The problem addressed is plastering of walls and ceilings during the construction of apartment and office buildings. The concrete walls are to be covered by a smooth layer of plaster, a few millimetres thick. The workspace is structured enough that map generation, navigation and motion planning can be performed autonomously. The robot must reliably avoid plastering windows and doors. Typical requirements on the navigation accuracy are standard deviations no greater than $1 \mathrm{~cm}$.

Two prototypes are described. For navigation the robot uses a range measuring sensors scanning in one (or two) planes. The observations are used to create, and continuously update, a mainly planar map with some vertical information - i.e. door and window positions. The map generation and sensor fusion algorithms are based on a Bayesian association algorithm and an extended Kalman
filter.

Tests have been successfully performed at an actual construction site. The time used for plastering the walls and ceiling in a room is expected to be less than 50\% of that required by manual work. The amount of plaster used is also greatly reduced due to more even spraying. However, the reasons for developing the robot is mainly
\end{abstract}

Keywords: Plastering robot, Autonomous mobile robot, Construction robot, Robot navigation, Multi sensor fusion, Extended Kalman filter, Hough/Radon transform,

\section{Introduction}

This paper describes an autonomous mobile robot system for indoor surface operations at a building construction site. The sensors, mechanical design and algorithms for compared for two different prototypes.
.
The task for which the robots are designed is the spray plastering of concrete walls and ceilings during the construction of apartment/office buildings. During this with a concrete walls and ceilings are to be covered smooth surface. Typical thick layer of plaster to create a accuracy is $1 \mathrm{~cm}$ standar requirements for the navigation test case for an autonard deviation. This is a very good and the environment is robot: The task is well defined tered. It is also a task which weared and reasonably uncluttion workers thus automation.

To accomplish the task the robot has to autonomously measure the size of the room and the positions of doors and windows. While spraying it has to continue to sense it's environment to update it's position estimate to fulfil the navigation requirements. Of course, it also has to avoid spraying doors and windows.

In addition to dead reckoning using axis encoders three sensing systems have been evaluated for the robot: $A$ sheet of light range camera, a scanning range measuring laser and a camera vision system. The latter used only as a complement to the range measuring laser.

Two main prototypes are employed: The first used linear actuators, pneumatic cylinders and one continuous revolute joint to steer the spray gun, see fig. 2 and 1 . In the second prototype, currently under construction, a Motoman robot arm, fig. 3, replaces this setup. In both
cases a tricycle mobile base is used.

The first prototype has been used in successful tests at an actual construction site in Skellefteå in northern Sweden. While the tests were successful there were some reliability problems, both software and hardware. The new prototype aims to solve these problems and bring the robot to a point were the development can be continued by a
commercial company.

References [1], [2] and [3] are useful background textbooks and [5] and [8] are useful papers when studying
the navigation system used here. 


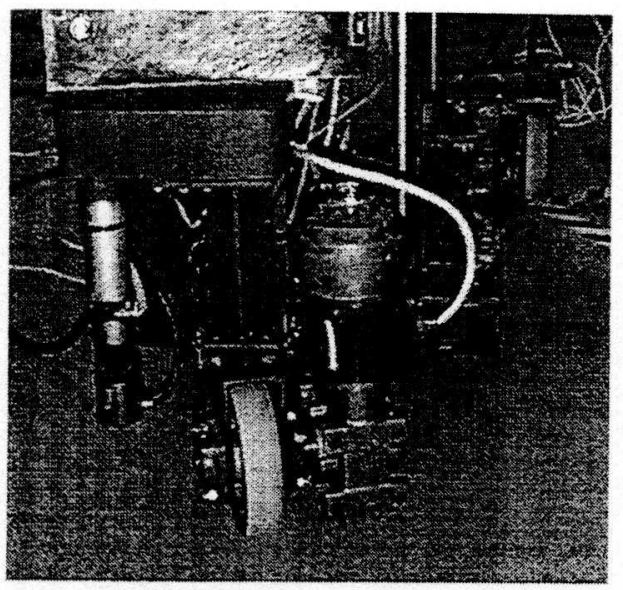

Fig. 1 The wheel placement on the first prototype.

\section{Robot and system overview}

Automatic plastering requires three components:

- A sensing system that measures the environment and plans the operation.

- A mechanical arm or lift to move the spray gun appropriately.

- A mobile platform to move the robot arm to the desired position, allowing it to work on both small and large rooms.

The mechanical component systems are described in this section, with examples of different approaches tested. There is a trade-off between the different subsystems. A more versatile arm requires a simpler less agile mobile platform than a simpler less agile arm. A mobile platform with poorly defined kinematics will require a more powerful navigation system.

\subsection{The robot system}

The system is divided into the following mechanical, sensory and computing parts:

- The mobile robot base with three wheels.

- Prototype 1: The three wheeled robot base has one steerable front wheel with traction and two rear wheels, one with traction. The rear wheels can be rotated to three different positions allowing the robot to manoeuvre in three different modes, as a car with a steering front wheel, rotating on the spot and crabbing sideways.

- Prototype 2: The three wheeled robot base has one steerable front wheel with traction and two rear wheel without traction.

- The robot arm
- Prototype 1: The spray gun is mounted in a special spray assembly moved by two linear cartesian actuators. This setup cannot compensate for heading errors or position errors perpendicular to the wall. Therefore the rotating and crabbing modes of the robot base are needed

- Prototype 2: The spray gun is mounted on a 6 DOF industrial robot arm.

- The navigation sensor, a range measuring sensor capable of looking in all directions, but only in one plane. Early version of prototype 1 used a sheet-oflight range camera mounted on a rotating base. Later this was replaced by a scanning range measuring laser.

- The door/window sensor.

- Prototype 1: A camera or a range camera is used to detect the upper and lower bounds of openings in the wall. This sensor can move with the spray gun.

- Prototype 2: Instead of using the camera, which was inherited from the earliest prototype which also used it for all the navigation, this version tilts the laser to get vertical rangescans of the interesting parts of the wall. Interesting parts are those were the horizontal scan showed an opening.

- The navigation system, used to create a map of the environment and to estimate the robot position relative to said environment.

- The robot control system used to control the motion of the robot relative to the environment with centimetre accuracy.

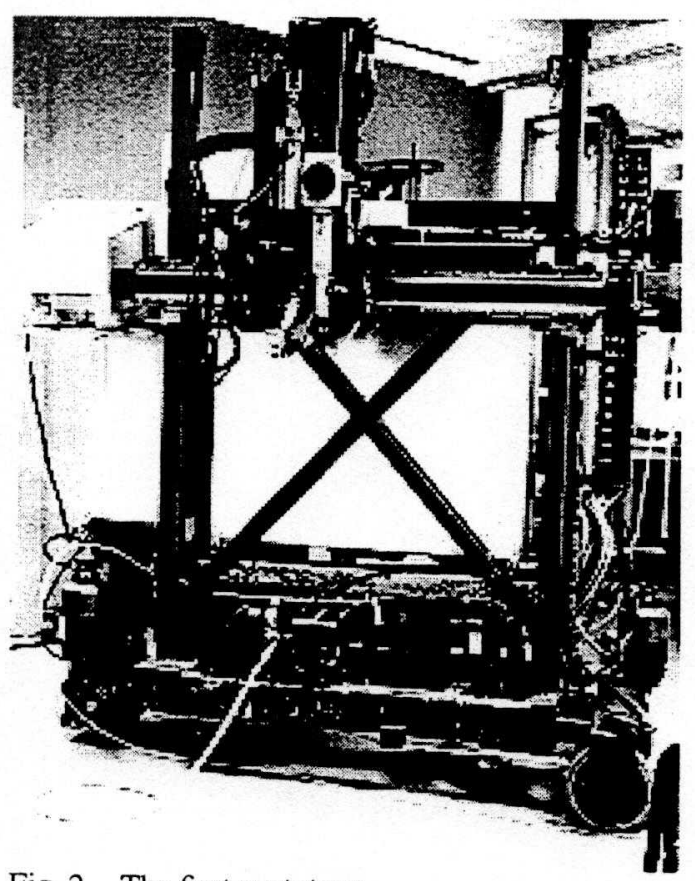

Fig. 2 The first prototype. 


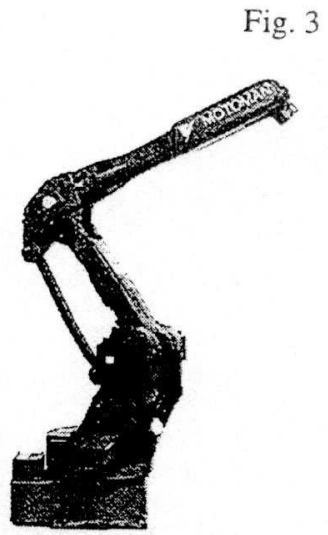

The second prototype uses a Motoman Sk6 robot arm to move the spray gun. The more flexible motion of the articulated robot arm compared to the $2 \mathrm{D}$ cartesian arm lowers the amount of motion corrections that the mobile platform has to perform.

- The plastering system. Plans and executes the plastering task as a sequence of robot motions.

- The security system. Collision and error detection and watchdog emergency stop. Currently quite limited but would be greatly expanded in a production system.

\subsection{The operating pattern}

The robot will work on a construction site were the floor has been finished and levelled and the concrete walls have been raised. Other walls (not made of concrete) have usually not been raised at this stage but some outer walls might be temporarily covered by plastic or boards. Plaster is supplied through a tube from a stationary pump at a pressure of more than 100 atmospheres. Electricity is also supplied using a cable.

To plaster a room the robot first has to measure the shape and size of the room and the position of door, windows and other large openings in the walls. After the measurement phase is finished the robot plans the plastering pattern, allowing the operator to specify whether any walls are to be left unplastered. The map and the plastering plan is displayed graphically for the operator. Finally the robot will autonomously move around the room spraying the walls and ceilings without further human intervention.

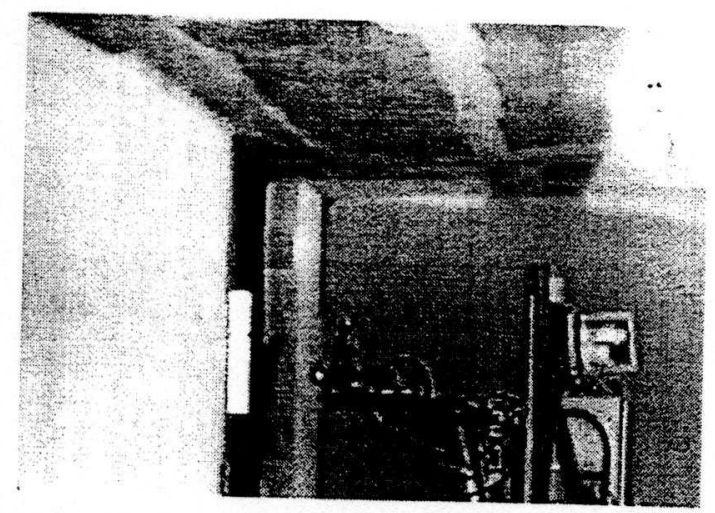

Fig. 4 The first prototype plastering the ceiling.

\section{Range measurements and automatic evaluation}

Two range measuring systems have been tested, a sheet-oflight range camera and a scanning range measuring laser Most of this section covers the laser as it is the best instrument for this type of applications. The range camera was found to be insufficient at the long ranges and with the difficult lighting conditions that exist in this application. For more information on how the range camera was used see [4] and 6.1. The range measuring sensor is mounted on top of the robot and is thus capable of looking in all directions and at such a level that it will see both door and window openings.

\subsection{The scanning range measuring laser}

The range measuring laser is an Accurange 3000. It allows the user to make a number of trade-offs between range, sampling frequency and accuracy. We have chosen sampling frequency of about $1900 \mathrm{~Hz}$ giving roughly 500 measurements per revolution of the scanner which rotates at a little less than $4 \mathrm{~Hz}$. The maximum range is 23 meters and each measurement usually have a standard deviation of less than one centimetre.

The scanner used allow measurements over 360 degrees in the horizontal plane. The laser is mounted $180 \mathrm{~cm}$ over the floor making all normal doors and windows visible to it. The entire laser scanner can also be tilted 90 degrees to measure a vertical scan. This is used to measure the upper and lower edges of the windows and doors detected in the horizontal scan. Earlier versions of the robot used a CCD camera for this, using the information from the range scan to cue the image processing algorithms.

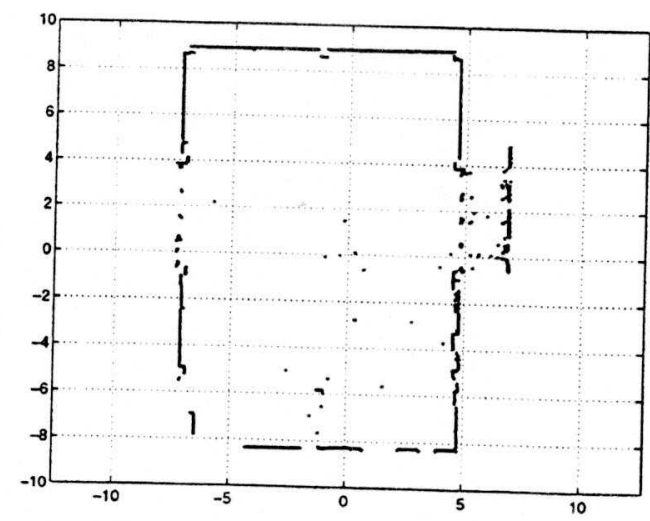

Fig. 5 A rangescan taken by the Accurange 3000 in the robotics laboratory at a height of $180 \mathrm{~cm}$. The scan consists of about 1200 measurements measured in 0.3 seconds. 


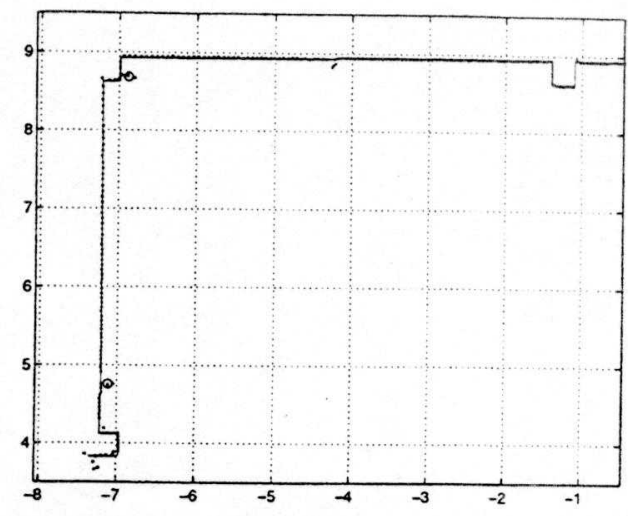

Fig. 6 A zoom in of the upper left corner. The laser measurements are shown as dots. The circles and lines are hand measured "ground truth" to show the absolute accuracy.

\subsection{Examples of measurements}

The capabilities of the laser is best shown by example. A full scan taken in the robotics lab is shown in fig. 5. Hand measurements were made for the upper left corner to establish a "ground truth" to compare the measurements with, fig. 6. More detailed zoom ins are shown in fig. 7 and fig. 8. The differences between the ground truth and the measurements are small enough that it cannot be established whether they are laser calibration errors or errors in the ground truth.

\subsection{The range-weighted Hough transform (RWHT)}

To find the walls of the room the range-weighted Hough transform is used, see [5]. The RWHT is essentially a two dimensional histogram to find the walls which have the

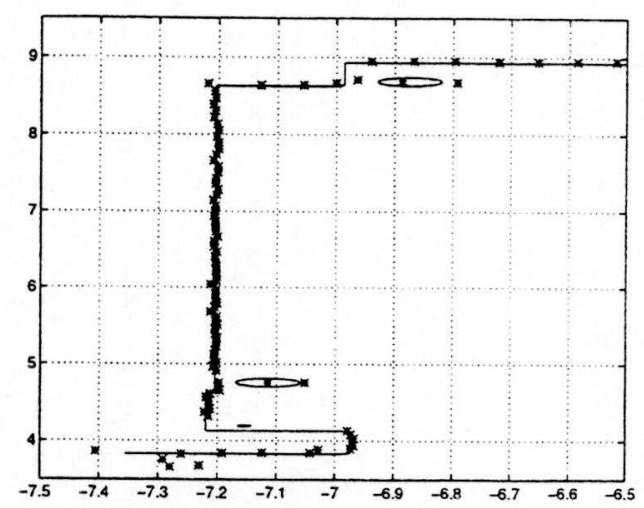

Fig. 7 A zoom in of the left wall. Note the different vertical and horizontal scales.
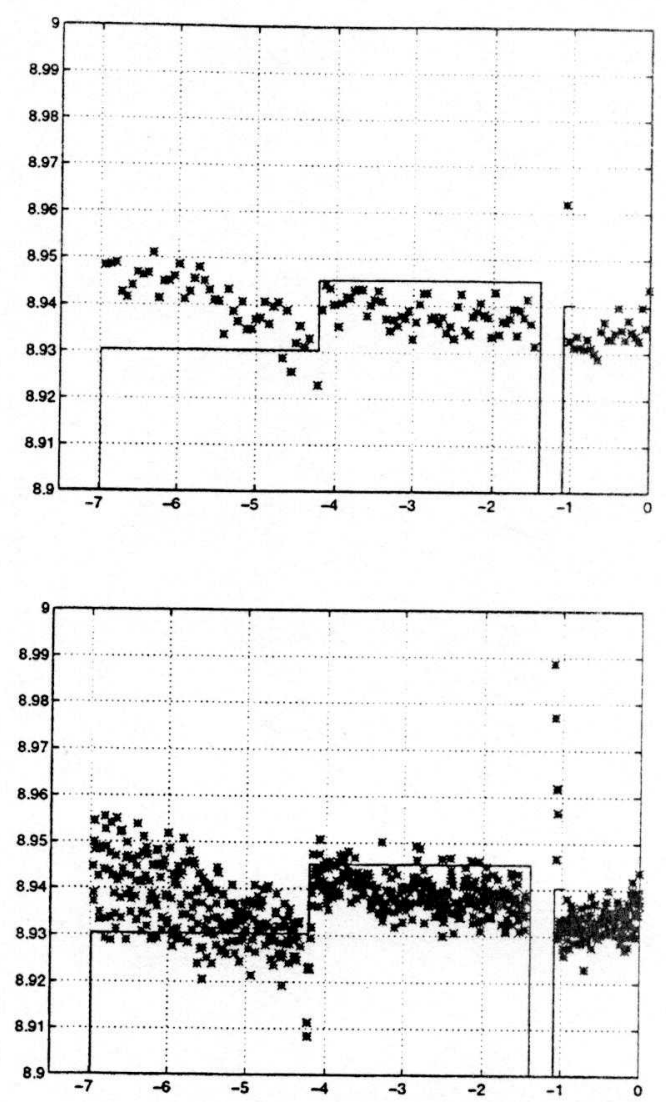

Fig. 8 An extreme zoom in of the left part of the top wall. Note the very different vertical and horizontal scales. The upper plot shows one measurement scan and the lower plot show seven overlayed scans. The $1.5 \mathrm{~cm}$ discontinuity in the wall is clearly visible in the measurements.

most measurements "supporting" it. Each measurement is weighted by the range to compensate for the range dependent sampling density of polar measurements. The Hough transform is a very robust method for extracting straight lines from range scans and can easily handle scans much more cluttered than those encountered in this application. The high information content in a range scan as compared to a conventional camera image allows it to be used in real time even on a relatively slow computer (Intel 486).

\subsection{Extracting landmarks and estimating their parameters}

For the robot to perform it's task it needs to know the positions of the walls of the room relative to itself, and also to know the positions and sizes of any windows or doors in these walls. For a human observer both these are clearly 
visible in the range scans and they can also be found in a straightforward way using the RWHT. The landmark observation process can be outlined as:

1 Preprocess the rangescan, including geometric compensation for motion while the scan was made.

2 Compute the RWHT and find the largest peak, which corresponds to the longest visible wall.

3 Match measurements to the parameters from the RWHT peak (which is only a rough estimate of the equation of the wall).

4 Use a least variance estimator on the matched measurements to get a better estimate of each wall.

5 Find any openings (doors/windows) by searching for jump edges or areas with out-of-range measurements. Their position is estimated from the jump edge position. Currently no attempt are made to actually measure the orthogonal edge of the wall.

6 Remove all matched points from the scan.

7 If not finished, repeat from point 2.

While the Hough transform is usually done with about 400 measurements per scan, the scan itself might contain more measurements. These are then used for the edge detection and least variance approximation which is less time consuming than the RWHT.

\section{Map generation and navigation}

The mapping and navigation system uses a range sensor scanning in a plane to acquire two dimensional measurements of the environment and wheel axis encoders to accumulate motion estimates. These measurements are used to create a map of the environment and to estimate the robots position relative to this map (actually, the maps position relative to the robot).

The range sensor is mounted at such a height that it will see all kinds of normally occurring openings (windows, doors etc.) in the walls. It cannot distinguish between for example doors and windows as it only measures in one plane. The first prototype had a second sensor: a camera mounted near the spray gun on the robotic arm. This camera provided the robot with the measurements needed to determine the upper and lower edges of openings in the walls. In the second prototype the scanning laser can tilt 90 degrees thus allowing it to observe the upper and lower edges of the openings. Note that a full 3D scan is not needed as we have prior knowledge about the environment - the openings are rectangular.

\subsection{The environment in robot coordinates}

The navigation system uses a planar model of the environment. Vertical information, window and door heights, are treated separately as they are uncorrelated with the robot position and the rest of the map. The map is given in coor- dinates relative to the robot, as no other natural origin exists in the general case. It contains two kinds of landmarks: walls, described as infinite lines, and endpoints for segments of the walls.

The coordinates for the walls are described by the distance $d$ and angle $\gamma$ from the robot to the point on the wall closest to the robot. The endpoints of wall segments are given as distances from this point parallel to the wall. Thus the state vector describing the environment will look like this:

$$
X=\left[\begin{array}{lllllllll}
d_{1} & \gamma_{1} & s_{11} & s_{12} & \ldots & d_{2} & \gamma_{2} & s_{21} & \ldots
\end{array}\right]^{T}
$$

where $d_{i}$ is the orthogonal distance from the robot to wall $i$ and $\gamma_{i}$ is the corresponding angle. $s_{i j}$ is the offset to edge $j$ on wall $i$, see fig. 9 .

When the robot moves the state vector is updated as:

$$
\begin{aligned}
d_{i}\left(t_{k}\right)=d_{i}\left(t_{k-1}\right) & -p_{x}\left(t_{k}\right) \cos \left(\gamma_{i}\left(t_{k-1}\right)\right)- \\
& -p_{y}\left(t_{k}\right) \sin \left(\gamma_{i}\left(t_{k-1}\right)\right) \\
\gamma_{i}\left(t_{k}\right)=\gamma_{i}\left(t_{k-1}\right) & -p_{\theta}\left(t_{k}\right) \\
s_{i j}\left(t_{k}\right)=s_{i j}\left(t_{k-1}\right) & +p_{x}\left(t_{k}\right) \sin \left(\gamma_{i}\left(t_{k-1}\right)\right)- \\
& -p_{y}\left(t_{k}\right) \cos \left(\gamma_{i}\left(t_{k-1}\right)\right)
\end{aligned}
$$

where the robot translation and rotation relative to the previous position is given as $\left(p_{x}, p_{y}, p_{\theta}\right)$.

The estimate of the environment is a state vector estimate $\hat{X}$ with covariance $\Sigma$. Note that the model usually only contains a subset of the full environment description, and that the dimension will increase as more of the world is observed.

\subsection{The observation model}

The range measurements can be used to directly observe the parameters in the state vector using the Range

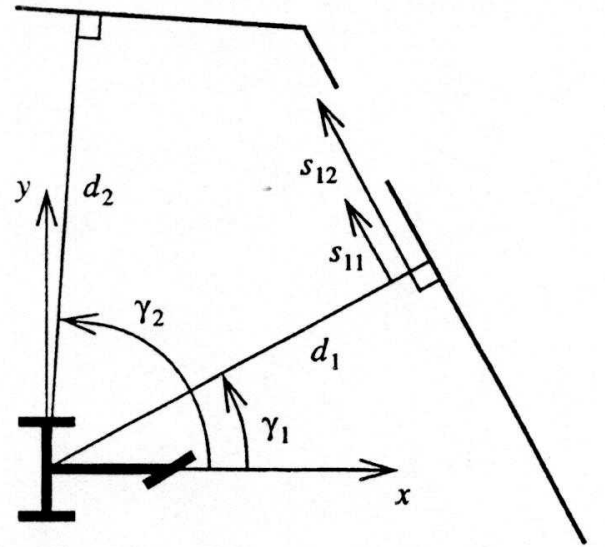

Fig. 9 The robot relative coordinates used to describe the position of walls $\left(d_{i}, \gamma_{i}\right)$ and openings at $s_{i j}$ along the wall. 
Weighted Hough Transform (RWHT) and a robust least variance estimation. The edges and endpoints are extracted separately. In the equations below the observations are indicated by an overline, i.e.

$$
\bar{d}=d+\text { Noise } \quad \bar{\gamma}=\gamma+\text { Noise }
$$

The endpoints can be observed as jump edges in the range image. The actual observation is the angle $\beta_{i j}$.

$$
\bar{\beta}=\gamma+\operatorname{atan} \frac{s}{d}+\text { Noise }
$$

In the case of the wall observations the variance and correlations for the two parameters are given by the least variance estimator. For the edge detection the variance is given by the angular resolution. All uncertainties are modelled as white Gaussian zero mean noise. Apart from the correlation between angle and distance the observations are assumed to be uncorrelated with each other and over time. This results in the following observation vector $Z$ and associated covariance matrix $S$.

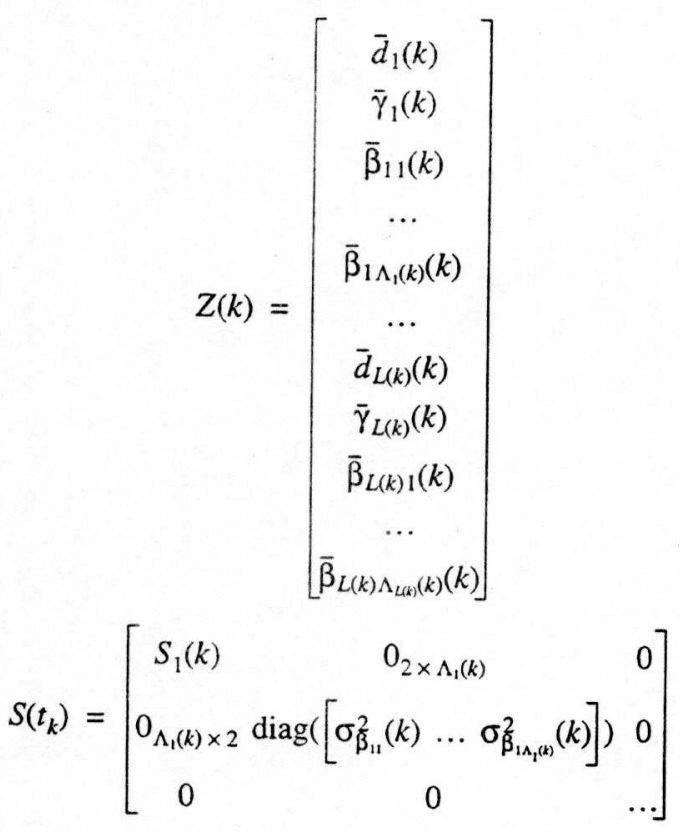

Where $L(k)$ is the number of observed walls and $\Lambda_{i}(k)$ is the number of observed edges for wall $i$ at time $k$.

\subsection{Association likelihoods and probabilities}

Thus we have three vectors of landmarks.

1 The full model $(X)$, which has an unknown (possibly infinite) length.

2 The latest observation $(Z, S)$ which is a subset of the full model. The identity of the observations are not known.
3 The map $(\hat{X}, \Sigma)$ which is a subset of the full model, possibly intersecting the latest observation.

The full model is only a concept used for deriving the equations and is not present in the solution.

The objective of this section is to find which, if any, of the observed landmarks are observations of landmarks already in the map and which are new. An important point to note is that we do not have to make an decision! It's better to ignore uncertain observations than to act incorrectly upon them. Therefore an approximate probability is calculated for each hypothesis and a threshold is applied to the most probable hypothesis. A more detailed description of the principles of the navigation system is given in [8].

\subsection{Updating the estimate}

When the identity of the observations are established they can be used to update the information in the map. This is done using an extended Kalman filter (EKF). Note that when new landmarks are observed, the map will grow and the state vector in the EKF will be extended. Motion information from encoders in the wheels are used for the prediction step in the EKF using the motion update equation (2). More details are given in [8].

\section{Control and execution}

\subsection{Trajectory following and positioning.}

The control of the robot is based on the idea of pointing the steering wheel at a point on the desired trajectory slightly in front of the steering wheel. When reversing an imaginary steering wheel behind the robot is used. However, the important target is not the robot motion, but the motion of the spray line on the wall (especially during horizontal plastering where the robot moves while it plasters). Therefore the control signal is calculated for an imaginary robot positioned at that point, and then the control of the real robot is calculated from this. As the robot is non-holonomic there are some restrictions on what is achievable using this method.

When attempting to achieve a desired position the first prototype used it's capability to rotate on the spot and to move sideways to correct initial positioning errors. The new prototype will not to this, but instead use the flexibility of the robotic arm to compensate for any remaining errors.

\subsection{Motion strategies with a $2 D$ cartesian manipulator.}

The first prototype used a 2D cartesian arm. This arm allowed the robot to reach all the way up and down along the wall and to reach both ends of the robot - necessary to 


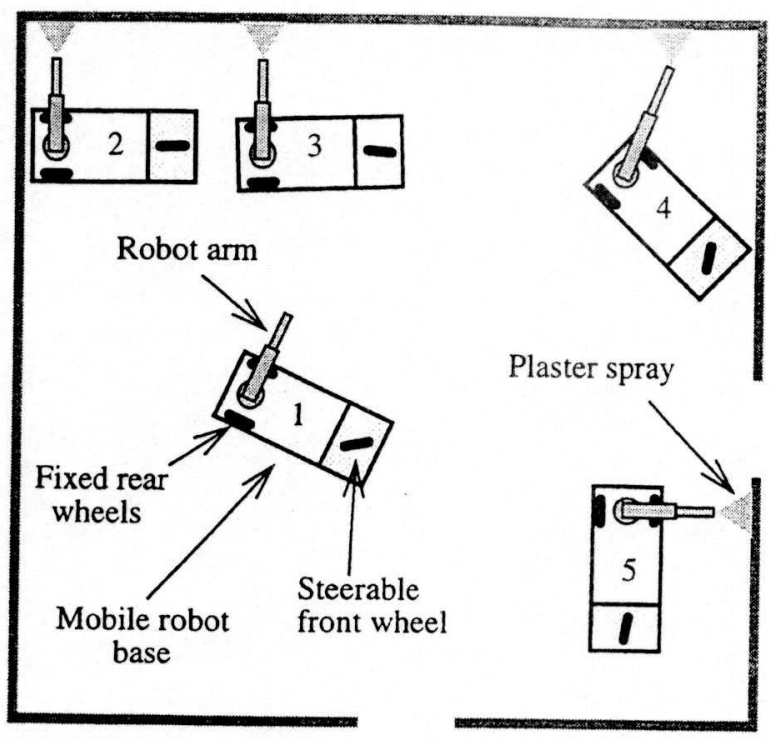

Fig. 10 Starting somewhere in the room (1) the robot creates a map from laser measurements. Starting at one wall (2) it plasters the wall $(3,5)$. The dexterity of the robot arm enables it to reach the wall even while turning (4). Small position and heading errors can be compensated by the arm.

reach into corners. It also had one continuous prismatic joint to direct the spray gun and binary prismatic joint to change to between ceiling, horizontal and vertical plastering. The robot base had the ability to move sideways and rotate on the spot in addition to the more normal carlike motion pattern. This flexibility was needed to manoeuvre efficiently in corners and to compensate for small remaining position and heading errors before plastering.

The robot plans the spraying pattern to cover the wall without spraying the windows. The limiting parameter, except for the position of the windows and length of the wall, is the useful spraying distance which limits the spray widths that are possible.

\subsection{Motion strategies with a 6-DOF articulated manipulator.}

The planning is essentially the same for both versions of the robot, see 5.2. However the execution is streamlined using an articulated arm. The typical motion pattern while plastering a room is shown in fig. 10. Unlike the 2D Cartesian arm, the articulated arm need not be long enough to reach both ends of the robot, as it can plaster even while the robot is in a turn, step (4) in fig. 10. Another advantage is that the ability to rotate on the spot and move sideways is no longer needed. Manoeuvring in corners is simplified as seen in fig. 10 and small positioning errors can be compen- sated using the robot arm rather than the non-holonomic robot base.

\section{Experimental results}

These results are from experiments with the first robot prototype. Experimental results for the second prototype were not available in time for this paper. Results are presented for navigation using both the range camera and the scanning range measuring laser. We do however concentrate on the scanning laser as that was the better sensor for this application

\subsection{Range camera navigation tests}

During the first tests a sheet-of-light range camera was used as the navigation sensor. This camera could be rotated to cover the entire plane. The measurement of the room was performed in two steps, first the dimensions of the room and the windows was measured from a position in the middle of the room, then the robot moved to the first wall and stopped in front of each window to observe it more accurately.

In laboratory tests the robot succeeded to estimate the positions of the windows on a wall with less than one centimetre standard deviation and the positioning of the robot relative to the wall was within a couple of centimetres. Much of the positioning errors were due to mechanical calibration errors in the zero angle of the steering wheel.

However, tests on a real construction site showed that the range camera was too sensitive to varying light conditions (sunshine). Especially longer ranges (more than 2-3 meters) caused problems both with detecting the laser line and large measurement errors due to calibration problems. Algorithms were developed, see [4], to automatically calibrate the range camera using the motion of the robot.

\subsection{Range measuring laser navigation tests}

During the tests below a scanning range measuring laser was used. Such a system is much less sensitive to light conditions and can measure at considerable larger ranges. It is however more expensive than a range camera and also requires moving parts.

The tests described below have been performed in a laboratory which is actually somewhat more cluttered than a typical room at a construction site. Tests at a construction site is discussed in 6.3. The laboratory is about 9 by 5 metres. In the experiments the robot went through the following steps, using the plastering program without any changes:

1 It always started in front of the same 5 meter long wall.

2 Initialization and map generation. 
3 Scanning the wall for windows/doors.

4 Plastering one horizontal length, requiring the robot to move twice along the wall (once in each direction).

5 Possibly repeating point 4 one or more times.

During these tests the following three parameters were evaluated. The statistics used are the standard and maximum deviation from the mean.

1 The repeatability of the distance from the wall.

2 The repeatability of the robot heading.

3 The stopping point, i.e. the positioning along the axis parallel to the wall.

The accuracy was evaluated using lines drawn by a pen mounted on the robot near the rear axis. Three sets of experiments were performed with three different operation patters.

1 Plastering multiple lengths on the wall. This experiment was performed exactly as outlined above, repeating the plastering 7 times for a total of 14 legs along the wall. Every leg followed directly after the preceding leg.

This experiments mainly tests the navigation along the wall.

2 Plastering multiple lengths on the wall, with the robot moved between each length. The robot was moved before starting each new length to introduce a position disturbance and to better test the positioning of the robot. This was repeated 4 times for a total 8 legs.

This experiments also tests the positioning before moving along the wall.

3 Restarting between each length. Here the robot was restarted, including a reinitialization, a new scan of the wall and several meters of manoeuvring, between each length. This was repeated 6 times for a total of 12 legs. This experiments tests the repeatability of the map generation/initialization.

The experiments revealed a calibration error which affects the accuracy. The laser is not properly aligned causing the shape of the room to be distorted. When the robot moves in the room the navigation system averages information from scans taken at different points. This removes most of the effect on the navigation repeatability. Therefore the robot was allowed to move along the wall ones after each initialization to properly observe the wall before the evaluation runs. The results are shown in table I. Without the initial observation run the errors would be larger. The scans shown in 3.1 where taken after this calibration error had been corrected.

To summarize the navigation results we note that the standard deviation of the attainable repeatability is about $0.5 \mathrm{~cm}$ and $1^{\circ}$. This result is both for the distance to the wall and for the stopping position.
Table I Experimental tests of repeatability

An evaluation of the repeatability of the system.

The speed of the robot was about $0.3 \mathrm{~m} / \mathrm{s}$.

After each restart of the navigation system the robot travelled along the wall ones to observe it before the evaluated runs. This was necessary due to a calibration error.

\begin{tabular}{|c|c|c|c|}
\hline Experiment & $\mathrm{N}$ & $\begin{array}{l}\text { Standard } \\
\text { deviation }\end{array}$ & $\begin{array}{l}\text { Maximum } \\
\text { Spread }\end{array}$ \\
\hline 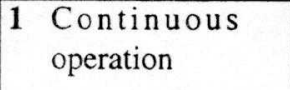 & 10 & $\begin{array}{c}0.3 \mathrm{~cm} \\
0.2^{\circ}\end{array}$ & $\begin{array}{l}1 \mathrm{~cm} \\
0.6^{\circ}\end{array}$ \\
\hline $\begin{array}{l}2 \text { Movement } \\
\text { between lengths }\end{array}$ & 7 & $\begin{array}{c}0.6 \mathrm{~cm} \\
1^{\circ}\end{array}$ & $\begin{array}{c}2 \mathrm{~cm} \\
3^{\circ}\end{array}$ \\
\hline \begin{tabular}{|ll}
3 & $\begin{array}{l}\text { Full restart } \\
\text { between lengths }\end{array}$
\end{tabular} & 6 & $\begin{array}{c}0.5 \mathrm{~cm} \\
1^{\circ}\end{array}$ & $\begin{array}{c}1.5 \mathrm{~cm} \\
3^{\circ}\end{array}$ \\
\hline Stopping position & 14 & $0.5 \mathrm{~cm}$ & $1.4 \mathrm{~cm}$ \\
\hline
\end{tabular}

\subsection{Autonomous plastering of an entire room}

To test the entire system, from sensor to actuator to the spray pattern on the wall, a demonstration was arranged at a construction site in Skellefteå. The demonstration showed the robot successfully plastering the walls as well as the ceilings of several rooms at the construction site.

\subsection{Error sources}

The error sources can be roughly divided into three main categories based upon which part of the system they originates from. They are mechanical errors, navigation errors and control errors.

\section{Mechanical error sources}

- Play in the wheels. The exact direction in which the wheels point is not known. There are play not only in the steering wheel direction, but also in the rear wheels directions. This usually causes an offset in the trajectory. This is expected to be a much smaller problem in the new prototype as the rear wheels will not be steerable.

- Play in the spray gun rotation causes the spray to hit the wall in the wrong position. With the spray gun mounted on an industrial robot arm this play should be greatly reduced.

- Calibration errors in the zero angle for the scanning laser causes navigation errors and an offset in the trajectory.

- Alignment errors in the laser mounting causes errors in the laser measurements that varies with the position of the robot. 


\section{Navigation errors}

- Errors due to the general uncertainty in measurements. These can be reduced using standard methods from signal processing and control theory.

- Association errors. These can result in single very large errors that are very difficult to model. This is an active area of research.

- Model errors can cause problems where the filter converges to the wrong parameter value. These can be everything from bad assumptions about the environment, i.e. the room isn't exactly rectangular, to discrepancies due to unmodelled mechanical misalignments. The latter is the cause of the initialization errors in the end of section 6.2 . In this case it can be solved by realigning or recalibrating the scanning laser.

\section{Control errors}

- Offsets caused by mechanical errors might cause steady state errors unless the offsets are modelled by the control law.

- Precise positioning using $\mathrm{AC}$ motors is difficult and the positioning of the robot is thus inexact. The new prototype has only DC motors. More advanced controllers can also be a solution if $\mathrm{AC}$ motors are preferred.

- Conflicting requirements on the non holonomic robot base. During plastering it is more important to keep a steady course over the entire length than to keep exactly the correct distance from the wall.

\subsection{Summary of error sources and experiments}

The results as given in table I with standard deviation of $0.5 \mathrm{~cm}$ and $1^{\circ}$ includes both navigation errors due to noise and control errors. Considering that no attempt has been made to make the robot kinematically precise the results should be considered surprisingly good, and sufficient for this task.

\section{Conclusions and future work}

The results show that $\mathrm{e}$ an autonomous mobile robot can be used for plastering entire rooms. An earlier version of the system was described in [6] and [7]. Some of the principles used in the navigation system can be found in [4], [5] and [8].

The accuracy is more than sufficient assuming that the calibration and mechanical problems are solved in the new prototype. The laser has already been tested with better calibration, see 3.1 .

There are many interesting extensions that are possible. The autonomy of the robot can be increased by adding further sensing capabilities. On line measurements of the spray patter using a sheet-of-light range camera would make the system considerably more robust and flex- ible. Using the full power of the navigation system could allow the robot to autonomously work on an entire level of an apartment building rather than in one room at a time. This last point do however put very large requirements on the autonomy of the robot.

The same system can be used for many other tasks. Especially the prototype using an articulated robot arm is very flexible and adaptable. Obvious other tasks are spray painting and grinding/polishing of walls. The latter might require a stronger robot arm than the one used. The same algorithms can be used in other robots for surface operations like spraying concrete on the walls of tunnels etc.

\section{Acknowledgements}

This work was supported by Arbetsmiljöfonden and Byggforskningsrådet with Byggergonomi Laboratorium (BELAB) in Stockholm as coordinator and in cooperation with DANEX AB, Skellefteå, Motoman Robotics AB, Torsås, Måleriutveckling, Nordsjö Nobel and Dalaspackel.

\section{References}

[1] B. D. O. Anderson, J. B. Moore, Optimal Filtering. Prentice Hall, 1979.

[2] S. Deans, The Radon Transform and some of its Applications. John Wiley \& Sons Inc., 1983.

[3] R. Duda, P. Hart, Pattern Classification and Scene Analysis. John Wiley \& Sons Inc., 1973.

[4] J. Forsberg, "Automatic calibration of a range camera using motion", Robot and Computer Vision, SPIE vol. 2354, pp. 261-270, Boston, 1994.

[5] J. Forsberg, U. Larsson, Å. Wernersson, “On Mobile Robot Navigation in Cluttered Rooms using the Range Weighted Hough Transform", IEEE Robotics and Automation Magazine, Special issue on Mobile Robots, 1995.

[6] J. Forsberg, D. Graff, Å. Wernersson, “An Autonomous Plastering Robot for Walls and Ceilings", IFAC Int. Conf. on Intelligent Autonomous Vehicles, Helsinki 1995.

[7] D. Graff, "Semiautonomous plastering robot for the building industry", Internal Report Luleå University of Technology, 1995.

[8] U. Larsson, J. Forsberg, A. Wernersson, "Mobile Robot Localization: Integrating Measurement from a Time-of-Flight Laser", IEEE Transactions on Industrial Electronics, special issue on Multisensor Fusion and Integration for Intelligent Systems, 1996. 